Journal of Computational and Applied Mechanics, Vol. 13, No. 1, (2018), pp. 5-14

DOI: $10.32973 /$ jcam.2018.002

\title{
ESTIMATION OF HEAT FLOW IN CIRCULAR BARS OF VARIABLE DIAMETER
}

\author{
István ECSEDi AND ATtila BAKsa \\ Institute of Applied Mechanics, University of Miskolc \\ H-3515 Miskolc-Egyetemváros, Hungary \\ mechecs@uni-miskolc.hu, mechab@uni-miskolc.hu
}

[Received: January 9, 2018; Accepted: May 15, 2018]

\begin{abstract}
Upper and lower bounds for the heat flow in nonhomogeneous circular bars of variable diameter are presented. The thermal properties may depend on the axial and radial coordinates, and the boundary conditions of the considered heat conduction problem do not depend on the polar angle. The analysed steady-state heat conduction problem is axisymmetric. Equations of Fourier's theory are used to formulate the thermal boundary value problem of heat conduction in nonhomogeneous circular bars with nonuniform crosssection. The computation of the heat flow is based on the concept of overall heat transfer coefficient. The derivation of bounding formulae for the overall heat transfer coefficient is based on a minimum principle and Schwarz's inequality. Six examples illustrate the applications of the derived upper and lower bound formulae how one can use to estimate the heat flow in a nonhomogeneous circular bar with nonuniform cross section.
\end{abstract}

Mathematical Subject Classification: 80A20, 80M30

Keywords: Heat flow, lower and upper bounds, nonhomogeneous, nonuniform circular bar

\section{INTRODUCTION}

The overall heat transfer coefficient in the steady-state heat conduction problem is an important structural property of a solid body in which the heat is flowing between its two separated parts of its boundary surfaces. The exact (strict) value of the overall heat transfer coefficient is known only with bodies of very simple shapes; therefore principles and methods are needed for creating lower and upper bounds to the numerical value of the overall heat transfer coefficient. From the higher temperature boundary part of body to the lower temperature boundary part of the body the process of heat flow is characterized by the overall heat transfer coefficient according to the equation $Q=\Lambda\left(T_{1}-T_{2}\right), T_{1}>T_{2}$ where $Q$ is the heat flow in unit time, $T_{1}$ and $T_{2}$ are given temperature and $\Lambda$ is the overall heat transfer coefficient. There are several papers which formulate upper and lower bounds for the heat flow in the case of steady-state heat conduction problems. In [1] the author examines the problem of planar heat conduction through an irregularly shaped body found as an inclusion in a perfectly insulating wall between two half-planes maintained at different temperatures. He obtains upper and lower bounds for the heat flow in terms of the temperature difference, conductivity and some global properties of the body. 
The presented method is based on the Schwarz's inequality. Paper 2 deals with the problem of determining the temperature distribution for steady-state heat conduction in a long cylindrical pipe. The author gives upper and lower bounds of the heat flow for the case of constant parameters describing the conductivity and density. In [3] a heat conduction problem in hollow three-dimensional body is considered and the author derives some inequality relations by the application of which lower and upper bounds may be obtained for the numerical value of the overall heat transfer coefficient. A linear problem of the steady-state heat conduction is studied in isotropic inhomogeneous hollow rigid bodies in 4. Applying Schwarz's inequality upper and lower bounds are derived for the overall heat conduction coefficient. Some mean value formula and bounds on the thermal energy for the steady-state heat conduction in anisotropic three-dimensional body are proven in 5]. The upper and lower bounds for the heat flux are derived by the application of Schwarz's inequality, avoiding the application of the minimum principles of potential thermal energy and complementary heat flux energy which were developed by Wojnar $[6]$.

\section{Governing EQUATIOns}

Let us consider a bar in the form of body of rotation. In cylindrical coordinates $(r, \varphi, z)$ the domain under consideration is $z_{1} \leq z \leq z_{2}, 0 \leq r \leq R(z), 0 \leq \varphi \leq 2 \pi$ and the axis of the bar is taken as the axis $z$ (Figure 1). This body of rotation occupies the region $\bar{B}=B \cup \partial B$, where the inner points of $B$ are denoted by $B$ and the set of boundary points of $\bar{B}$ is denoted by $\partial B$. $\partial B$ is divided into three parts as $\partial B_{1}=A_{1}$, $\partial B_{2}=A_{2}$ and $\partial B_{3}$. It is obvious that $\partial B=\partial B_{2} \cup \partial B_{2} \cup \partial B_{3}$.

The boundary surface $\partial B_{i}(i=1,2,3)$ is defined as

$$
\begin{gathered}
\partial B_{3}=\left\{(r, \varphi, z) \mid r=R(z), z_{1} \leq z \leq z_{2}, 0 \leq \varphi \leq 2 \pi\right\}, \\
\partial B_{i}=\left\{(r, \varphi, z) \mid z=z_{i}, 0 \leq r \leq R_{i}, 0 \leq \varphi \leq 2 \pi\right\} \quad(i=1,2), \\
R_{1}=R\left(z_{1}\right), \quad R_{2}=R\left(z_{2}\right) .
\end{gathered}
$$

The temperature in the body is denoted by $T=T(r, \varphi, z)(r, \varphi, z) \in \bar{B}$ and $k=$ $k(r, z)(r, \varphi, z) \in \bar{B}$ denotes the thermal conductivity of the material of the nonuniform circular bar. The local heat transfer coefficient at cross section $z_{i}(i=1,2)$ is denoted by $h_{i}=h_{i}\left(r, z_{i}\right)(r, \varphi, z) \in \partial B_{i}(i=1,2)$.

There is no distributed heat source in $B$ and no heat flux across the boundary surface segment $\partial B_{3}$. The boundary surface segment $\partial B_{i}$ is subjected to convective heat exchange and "fluid" temperature $T_{i}(i=1,2)$. It is assumed that $T_{1}$ and $T_{2}$ are constants and $T_{1}>T_{2}$.

With Fourier's theory of heat conduction 7,9 it can be shown that under the conditions prescribed above the temperature field of a nonuniform circular bar can be obtained as

$$
T(r, z)=\left(T_{1}-T_{2}\right) \theta+T_{2},
$$




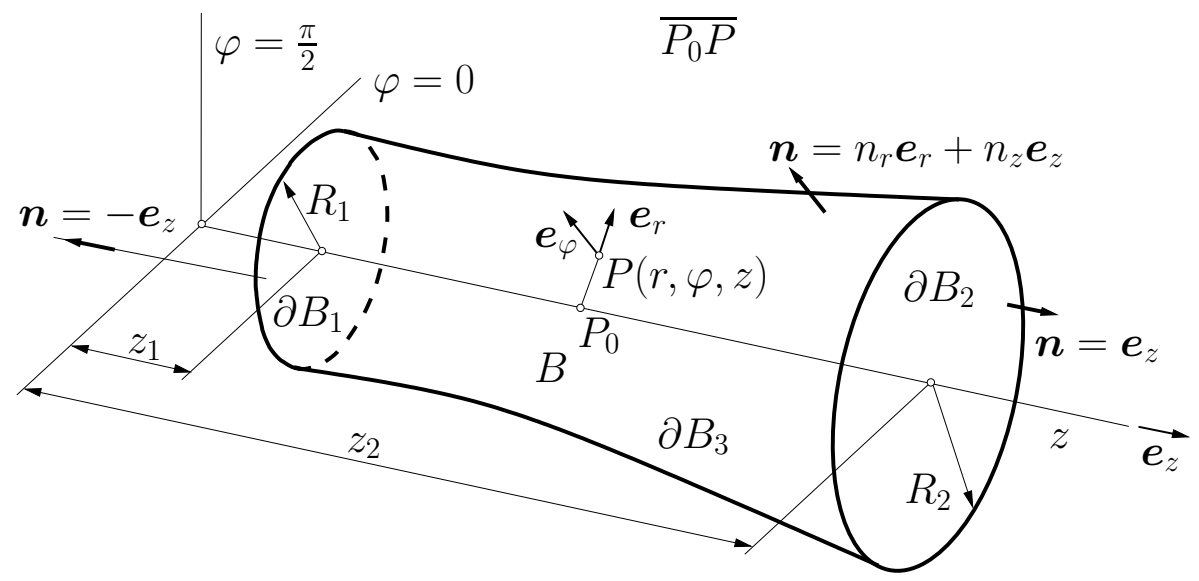

Figure 1. The body of rotation

where the function $\theta=\theta(r, z)$ is the solution to the next boundary value problem (Figure 1)

$$
\begin{gathered}
\nabla \cdot(k \nabla \theta)=0 \quad \text { in } B, \\
\boldsymbol{n} \cdot \nabla \theta=0 \quad \text { on } \quad \partial B_{3}, \\
k \boldsymbol{n} \cdot \nabla \theta+h_{1}(\theta-1)=0 \quad \text { on } \partial B_{1}, \\
k \boldsymbol{n} \cdot \nabla \theta+h_{2} \theta=0 \quad \text { on } \partial B_{2} .
\end{gathered}
$$

Here, the symbol $\nabla=\frac{\partial}{\partial r} \boldsymbol{e}_{r}+\frac{1}{r} \frac{\partial}{\partial \varphi} \boldsymbol{e}_{\varphi}+\frac{\partial}{\partial z} \boldsymbol{e}_{z}$ is the Hamilton-type vector differential operator in cylindrical coordinate frame, $\boldsymbol{n}$ is the unit outward normal vector on $\partial B$, dot between two vectors denotes their scalar product and $\boldsymbol{e}_{r}=\boldsymbol{e}_{r}(\varphi)$ is the unit vector in radial direction, $\boldsymbol{e}_{z}$ is the unit vector in axial direction and $\boldsymbol{e}_{\varphi}(\varphi)=\boldsymbol{e}_{z} \times \boldsymbol{e}_{r}(\varphi)$. The cross between two vectors denotes their vectorial product.

We note that the boundary value problem relating to the function $\theta=\theta(r, z)$ is axisymmetric and on the axis of revolution the following boundary condition can be obtained from the symmetry:

$$
\frac{\partial \theta}{\partial r}=0 \quad r=0, \quad 0 \leq z \leq L .
$$

Heat flows from the cross section $A_{1}$ through the circular bar of variable diameter to the cross section $A_{2}$. This process is characterized by the equation

$$
Q=\Lambda\left(T_{1}-T_{2}\right), \quad T_{1}>T_{2} .
$$

In equation (2.7) $\Lambda$ is a constant called the overall heat transfer coefficient and its value depends on the shape and the thermal properties of the nonuniform circular bar, while $Q$ denotes the heat conducted within unit of time through the end sections. Here, we note in the book by Carslaw and Jaeger 8. that the thermal resistance $\rho$ is defined by the equation $\rho \Lambda=1$. It is evident from 7,9$]$ that 


$$
\begin{array}{r}
Q=\int_{A_{1}} k \boldsymbol{n} \cdot \nabla T \mathrm{~d} A=-\int_{A_{2}} k \boldsymbol{n} \cdot \nabla T \mathrm{~d} A=\left(T_{1}-T_{2}\right) \int_{A_{1}} k \boldsymbol{n} \cdot \nabla \theta \mathrm{d} A= \\
\quad\left(T_{2}-T_{1}\right) \int_{A_{2}} k \boldsymbol{n} \cdot \nabla \theta \mathrm{d} A .
\end{array}
$$

Starting from the equation

$$
\nabla \cdot(\theta k \nabla \theta)=k|\nabla \theta|^{2}+\theta \nabla \cdot(k \nabla \theta)
$$

by integration and by the application of the Gaussian theorem of integral transform and equations $2.2,2.3), 2.4,2.5$ we obtain

$$
\begin{aligned}
& 0= \int_{B} \nabla \cdot(k \theta \nabla \theta) \mathrm{d} B-\int_{B} k|\nabla \theta|^{2} \mathrm{~d} B=\int_{\partial B} \theta k \boldsymbol{n} \cdot \nabla \theta \mathrm{d} \partial B-\int_{B} k|\nabla \theta|^{2} \mathrm{~d} B= \\
& \int_{A_{1}} \theta k \boldsymbol{n} \cdot \nabla \theta \mathrm{d} A+\int_{A_{2}} \theta k \boldsymbol{n} \cdot \nabla \theta \mathrm{d} A-\int_{B} k|\nabla \theta|^{2} \mathrm{~d} B= \\
&-\left\{\int_{B} k|\nabla \theta|^{2} \mathrm{~d} B+\int_{A_{1}} \frac{k^{2}}{h_{1}}(\boldsymbol{n} \cdot \nabla \theta)^{2} \mathrm{~d} A+\int_{A_{2}} \frac{k^{2}}{h_{2}}(\boldsymbol{n} \cdot \nabla \theta)^{2} \mathrm{~d} A\right\}+\int_{A_{1}} k \boldsymbol{n} \cdot \nabla \theta \mathrm{d} A .
\end{aligned}
$$

The combination of formulae (2.7) and (2.8) with equation 2.10 yields

$$
\Lambda=\int_{B} k|\nabla \theta|^{2} \mathrm{~d} B+\sum_{i=1}^{2} \int_{A_{i}} \frac{k^{2}}{h_{i}}(\boldsymbol{n} \cdot \nabla \theta)^{2} \mathrm{~d} A .
$$

It follows from equation 2.11 that $\Lambda>0$.

The primary purpose of this paper is to derive such inequality relations using which lower and upper bounds may be found for $\Lambda$. The exact value of the overall heat transfer coefficient $\Lambda$ may be given only with the knowledge of the solution to the boundary value problem defined by equations $(2.2),(2.3),(2.4)$ and $(2.5)$. The solution of the explicit form of the boundary value problem formulated in equations 2.2), 2.3, 2.4 and 2.5 is known only for bodies $B$ of very simple shapes 7,9 , therefore identifying principles and methods for producing lower and upper bounds to the numerical value of $\Lambda$ is a topic of great significance. On the other hand, some of the bounding formulae of $\Lambda$ may be the theoretical framework for the different types of finite element formulation of the heat conduction problem described by the equations (2.2), (2.3), 2.4) and (2.5).

\section{UPPER BOUND}

We introduce the symbol $E[\phi]$ by the definition

$$
E[\phi]=\int_{B} k|\nabla \phi|^{2} \mathrm{~d} B+\int_{A_{1}} h_{1}(\phi-1)^{2} \mathrm{~d} A+\int_{A_{2}} h_{2} \phi^{2} \mathrm{~d} A,
$$


where $\phi=\phi(r, \varphi, z)$ is a function for which the integrals appear in (3.1) exist and where they have finite values.

Theorem 1. Let $F=F(r, z)$ be continuous in the domain $\bar{M}$ and in the domain $M$ at least once continuously differentiable, otherwise an arbitrary function of $r$ and $z$. The inequality relation

$$
\Lambda \leq E[F]
$$

is valid.

The domain $\bar{M}=M \cup \partial M$ is the meridian section of the body of rotation $\bar{B}$. This means that $\bar{M}=\left\{(r, z) \mid 0 \leq r \leq R(z), z_{1} \leq z \leq z_{2}\right\}$ and $\partial M=\partial M_{1} \cup \partial M_{2} \cup \partial M_{3} \cup$ $\partial M_{4}$, where $\partial M_{1}=\left\{(r, z) \mid z=z_{1}, 0 \leq r \leq R_{1}\right\}, \partial M_{2}=\left\{(r, z) \mid z=z_{2}, 0 \leq r \leq R_{2}\right\}$, $\partial M_{3}=\left\{(r, z) \mid r=R(z), z_{1} \leq z \leq z_{2}\right\}$ and $\partial M_{4}=\left\{(r, z) \mid r=0, z_{1} \leq z \leq z_{2}\right\}$.

Proof. Consider the function

$$
\eta(r, z)=\theta(r, z)-F(r, z) .
$$

Using the expressions of $E[F]$ and $E[\phi]$ we obtain

$$
\begin{aligned}
E[F]=E[\theta]+\int_{B} k|\nabla \eta|^{2} \mathrm{~d} B+\sum_{i=1}^{2} \int_{A_{i}} h_{i} \eta^{2} \mathrm{~d} A+2\left\{\int_{B} k \nabla \theta \cdot \nabla \eta \mathrm{d} B+\right. \\
\left.\qquad \int_{A_{1}} h_{1}(\theta-1) \eta \mathrm{d} A+\int_{A_{2}} h_{2} \theta \eta \mathrm{d} A\right\} .
\end{aligned}
$$

By lengthy, but elementary calculations which involve the application of the derivation of product function and Gaussian theorem of integral transformation, the following relationship may be deduced

$$
\begin{aligned}
\int_{B} k \nabla \theta \cdot \nabla \eta \mathrm{d} B+\int_{A_{1}} h_{1}(\theta-1) \eta \mathrm{d} A+\int_{A_{2}} h_{2} \theta \eta \mathrm{d} A= \\
\int_{\partial B} k \eta \boldsymbol{n} \cdot \nabla \theta \mathrm{d} \partial B-\int_{B} \eta \nabla \cdot(k \nabla \theta) \mathrm{d} B+\int_{A_{1}} h(\theta-1) \mathrm{d} A+\int_{A_{2}} h_{2} \theta \eta \mathrm{d} A= \\
\int_{A_{1}} \eta\left[k \boldsymbol{n} \cdot \nabla \theta+h_{1}(\theta-1)\right] \mathrm{d} A+\int_{A_{2}} \eta\left[k \boldsymbol{n} \cdot \nabla \theta+h_{2} \theta\right] \mathrm{d} A=0 .
\end{aligned}
$$

The combination of equation (3.4) with equation (3.5) leads to inequality relation 3.2 . From the demonstration it follows that equality in 3.2 can be reached only if $F=\theta$.

\section{LOWER BOUND}

Theorem 2. In $\bar{B}$ the continuous vector field $\boldsymbol{b}=\boldsymbol{b}(r, \varphi, z)$ differing from the identically zero vector should satisfy the differential equation

$$
\nabla \cdot \boldsymbol{b}=0 \quad \text { in } \quad B
$$


and the boundary condition

$$
\boldsymbol{n} \cdot \boldsymbol{b}=0 \quad \text { on } \quad \partial B_{3} .
$$

The following inequality relation is valid

$$
\Lambda \geq \frac{\left(\int_{A_{1}} \boldsymbol{b} \cdot \boldsymbol{n} \mathrm{d} A\right)^{2}}{\int_{B} \frac{\boldsymbol{b}^{2}}{k} \mathrm{~d} B+\int_{A_{1}} \frac{(\boldsymbol{b} \cdot \boldsymbol{n})^{2}}{h_{1}} \mathrm{~d} A+\int_{A_{2}} \frac{(\boldsymbol{b} \cdot \boldsymbol{n})^{2}}{h_{2}} \mathrm{~d} A} .
$$

Proof. Let us have

$$
D(\boldsymbol{e}, \boldsymbol{f})=\int_{B} \frac{\boldsymbol{e} \cdot \boldsymbol{f}}{k} \mathrm{~d} B+\sum_{i=1}^{2} \int_{A_{i}} \frac{(\boldsymbol{e} \cdot \boldsymbol{n})(\boldsymbol{f} \cdot \boldsymbol{n})}{h_{i}} \mathrm{~d} A,
$$

where $\boldsymbol{e}=\boldsymbol{e}(r, \varphi, z)$ and $\boldsymbol{f}=\boldsymbol{f}(r, \varphi, z)$ defined in $B$ are two arbitrary continuous vector fields. On the basis of the Schwarz inequality it may be written that

$$
D(k \nabla \theta, k \nabla \theta) D(\boldsymbol{b}, \boldsymbol{b}) \geq(D(k \nabla \theta, \boldsymbol{b}))^{2} .
$$

It can easily be understood that

$$
\Lambda=D(k \nabla \theta, k \nabla \theta)
$$

The relationship

$$
\begin{aligned}
& D(k \nabla \theta, \boldsymbol{b})=\int_{B} \nabla \theta \cdot \boldsymbol{b} \mathrm{d} B+\int_{A_{1}} \frac{k}{h_{1}}(\boldsymbol{n} \cdot \nabla \theta)(\boldsymbol{n} \cdot \boldsymbol{b}) \mathrm{d} A+ \\
& \int_{A_{2}} \frac{k}{h_{2}}(\boldsymbol{n} \cdot \nabla \theta)(\boldsymbol{n} \cdot \boldsymbol{b}) \mathrm{d} A=\int_{\partial B} \theta \boldsymbol{n} \cdot \boldsymbol{b} \mathrm{d} \partial B+\int_{A_{1}} \frac{k}{h_{1}}(\boldsymbol{n} \cdot \nabla \theta)(\boldsymbol{n} \cdot \boldsymbol{b}) \mathrm{d} A+ \\
& \int_{A_{2}} \frac{k}{h_{2}}(\boldsymbol{n} \cdot \nabla \theta)(\boldsymbol{n} \cdot \boldsymbol{b}) \mathrm{d} A-\int_{B} \theta \nabla \cdot \boldsymbol{b} \mathrm{d} B=\int_{A_{1}} \boldsymbol{n} \cdot \boldsymbol{b}\left(\theta+\frac{k}{h_{1}} \boldsymbol{n} \cdot \nabla \theta\right) \mathrm{d} A+ \\
& \int_{A_{2}} \boldsymbol{n} \cdot \boldsymbol{b}\left(\theta+\frac{k}{h_{2}} \boldsymbol{n} \cdot \nabla \theta\right) \mathrm{d} A=\int_{A_{1}} \boldsymbol{n} \cdot \boldsymbol{b} \mathrm{d} A
\end{aligned}
$$

further, inequality (4.5) and formula 4.6 by their combination directly yield the lower bound formula (4.3) to be proven. In deriving the relationship 4.7) the rule of differentiation of the product function as well as the Gaussian integration theorem, equations (2.4), 2.5), 4.1) and 4.2 have been applied.

By some discussion it may be pointed out that in relation 4.3 the sign of equality is valid only in the case when

$$
\boldsymbol{b}=\alpha k \nabla \theta
$$

where $\alpha$ differs from zero otherwise being an arbitrary real constant. 


\section{EXAMPLES}

5.1. Example for upper bound. We assume

$$
F(r, z)=C_{1} \int_{z_{1}}^{z} \frac{\mathrm{d} \zeta}{K(\zeta)}+C_{2},
$$

where

$$
\begin{gathered}
C_{1}=\frac{1}{I+\frac{1}{H_{1}}+\frac{1}{H_{2}}}, \quad C_{2}=\frac{1+\frac{1}{H_{2}}}{I+\frac{1}{H_{1}}+\frac{1}{H_{2}}}, \\
K(z)=\int_{0}^{R(z)} r k(r, z) \mathrm{d} r, \quad I=\int_{z_{1}}^{z_{2}} \frac{\mathrm{d} z}{K(z)}, \quad H_{i}=\int_{0}^{R_{i}} r h_{i}(r) \mathrm{d} r \quad(i=1,2) .
\end{gathered}
$$

Inserting the function given by formula (5.1) into inequality relation 3.2 we obtain

$$
\Lambda \leq \Lambda_{U}=2 \pi C_{1}=\frac{2 \pi}{I+\frac{1}{H_{1}}+\frac{1}{H_{2}}} .
$$

5.2. Example for lower bound. In order to get the lower bound for $\Lambda$, we use in (4.3) the divergence free vector field

$$
\boldsymbol{b}=\frac{1}{R^{2}}\left[\frac{r}{R} \frac{\mathrm{d} R}{\mathrm{~d} z} \boldsymbol{e}_{r}(\varphi)+\boldsymbol{e}_{z}\right]
$$

This vector field satisfies boundary condition 4.2 and the condition

$$
\boldsymbol{b} \cdot \boldsymbol{e}_{r}=0 \quad r=0, \quad z_{1} \leq z \leq z_{2} .
$$

We introduce the following function and constants

$$
\begin{gathered}
M_{1}(z)=\int_{0}^{R(z)} \frac{r^{3}}{k(r, z)} \mathrm{d} r, \quad M_{2}(z)=\int_{0}^{R(z)} \frac{r}{k(r, z)} \mathrm{d} r, \\
N_{1}=\int_{z_{1}}^{z_{2}} \frac{M_{1}}{(R(z))^{6}}\left(\frac{\mathrm{d} R}{\mathrm{~d} z}\right)^{2} \mathrm{~d} z, \quad N_{2}=\int_{z_{1}}^{z_{2}} \frac{M_{2}}{(R(z))^{4}} \mathrm{~d} z, \quad N=N_{1}+N_{2}, \\
\frac{1}{S_{i}}=\frac{1}{R_{i}^{4}} \int_{0}^{R_{i}} \frac{r \mathrm{~d} r}{h_{i}(r)} \quad(i=1,2) .
\end{gathered}
$$

Putting the vector field given by the formula (5.7) into inequality relation (4.3), we get

$$
\Lambda \geq \Lambda_{L}=\frac{\pi}{2\left(N+\frac{1}{S_{1}}+\frac{1}{S_{2}}\right)} .
$$


5.3. Example for circular bar with uniform cross section. Let us apply formulae (5.4) and (5.10) to the circular cylindrical bar. We assume that the thermal conductivity depends only on the axial coordinate $z$ and $h_{1}, h_{2}$ are constants. In this case the upper and lower bounds formulated in $(5.4)$ and $(5.10)$ give the same result, which is the exact value of $\Lambda$. The computations yield the following value of $\Lambda$ :

$$
\Lambda=\frac{c^{2} \pi}{\int_{0}^{L} \frac{\mathrm{d} z}{k(z)}+\frac{1}{h_{1}}+\frac{1}{h_{2}}} \quad z_{1}=0, \quad z_{2}=L .
$$

In equation 5.11 the constant $c$ is the radius of the considered circular bar, that is $R(z)=c, 0 \leq z \leq L$.

5.4. Example for homogeneous circular cone. In this section, we deal with the homogeneous conical bars. Setting $R(z)=a+b z$, where $a$ and $b$ are constants and $z_{1}=0, z_{2}=L$. We find, from (5.4) and (5.10)

$$
\Lambda_{U}=\frac{\pi}{\frac{L}{k a(a+b L)}+\frac{1}{h_{1} a^{2}}+\frac{1}{h_{2}(a+b L)^{2}}}, \quad \Lambda_{L}=\frac{\pi}{\frac{\left(1+b^{2} / 2\right) L}{k a(a+b L)}+\frac{1}{h_{1} a^{2}}+\frac{1}{h_{2}(a+b L)^{2}}} .
$$

It is assumed in equation 5.12 that $k, h_{1}$ and $h_{2}$ are constants.

In the case $h_{i} \rightarrow \infty$ at the end cross section $A_{i}$, the Robin type boundary condition will be replaced by the Dirichlet type boundary conditionmeaning that the cross section $A_{i}$ is subjected to constant temperature $T_{i}(i=1,2)$.

Putting in formula $5.12 h_{1}, h_{2} \rightarrow \infty$ we obtain

$$
\frac{\Lambda_{U}}{\Lambda_{L}}=1+\frac{b^{2}}{2} .
$$

which shows that there is a significant difference between $\Lambda_{U}$ and $\Lambda_{L}$ for sufficiently large values of $b$.

Upper and lower bounds for $\Lambda$ may be improved by means of the Rayleigh-Ritz method [10 and finite element method [11], which are based on minimizing (3.2) with respect to $F=F(r, z)$ and maximizing (4.3) with respect to $\boldsymbol{b}=\boldsymbol{b}(r, \varphi, z)$.

5.5. Example for nonhomogeneous circular cylindrical bar of uniform cross section. Let $c$ be the radius of the boundary circle of the considered bar. The material properties are functions of the radial coordinate $r$. It is assumed that

$$
k(r)=k_{0} r, \quad h_{i}(r)=h_{0 i} r, \quad(i=1,2), z_{1}=0, z_{2}=L .
$$

Let $\Lambda_{0}$ be defined as

$$
\Lambda_{0}=\frac{c^{3} \pi}{\frac{L}{k_{0}}+\frac{1}{h_{01}}+\frac{1}{h_{02}}} .
$$

From the bounding formulae (5.4) and 5 the following result can be derived

$$
\lambda_{L}=\frac{\Lambda_{L}}{\Lambda_{0}}=\frac{1}{2} \leq \lambda=\frac{\Lambda}{\Lambda_{0}} \leq \lambda_{U}=\frac{\Lambda_{U}}{\Lambda_{0}}=\frac{2}{3} .
$$


Denote the mean value of $\lambda_{L}$ and $\lambda_{U} \bar{\lambda}=0.5\left(\lambda_{U}+\lambda_{L}\right)$. It is evident that

$$
|\lambda-\bar{\lambda}| \leq \frac{1}{12}
$$

5.6. Example for functionally graded circular cone. The points of the meridian section of a circular cone are given by the prescription

$$
\begin{aligned}
& \bar{M}=\left\{(r, z) \mid 0 \leq r \leq a z, z_{1} \leq z \leq z_{2}\right\} \quad \text { and } \partial M=\partial M_{1} \cup \partial M_{2} \cup \partial M_{3} \cup \partial M_{4}, \\
& \partial M_{1}=\left\{(r, z) \mid z=z_{1}, 0 \leq r \leq a z_{1}\right\}, \quad \partial M_{2}=\left\{(r, z) \mid z=z_{2}, 0 \leq r \leq a z_{2}\right\}, \\
& \partial M_{3}=\left\{(r, z) \mid r=a z, z_{1} \leq z \leq z_{2}\right\}, \quad \partial M_{4}=\left\{(r, z) \mid r=0, z_{1} \leq z \leq z_{2}\right\},
\end{aligned}
$$

The thermal properties are given functions of the radial coordinate according to equations

$$
k(r)=k_{0} \exp (\nu r), h_{1}(r)=h_{2}(r)=h_{0} \exp (\nu r),
$$

where $k_{0}, h_{0}$ and $\nu$ are material parameters. In the numerical example the following data are used: $a=0.5, z_{1}=0.8 \mathrm{~m}, z_{2}=3 \mathrm{~m}, k_{0}=100 \frac{\mathrm{W}}{\mathrm{mK}}, h_{0}=20 \frac{\mathrm{W}}{\mathrm{m}^{2} \mathrm{~K}}, \nu=0.5 \frac{1}{\mathrm{~m}}$.

Substitution of this data into equations (5.3), (5.4) and equations $(5.7,5.10$ gives

$$
\begin{gathered}
I=0.05769894569 \frac{\mathrm{K}}{\mathrm{W}}, H_{1}=1.830223478 \frac{\mathrm{W}}{\mathrm{K}}, H_{2}=37.65999967 \frac{\mathrm{W}}{\mathrm{K}}, \\
N_{1}=0.001731450850 \frac{\mathrm{K}}{\mathrm{W}}, N_{2}=0.0145366169 \frac{\mathrm{K}}{\mathrm{W}}, N=0.01626806776 \frac{\mathrm{K}}{\mathrm{W}}, \\
\frac{1}{S_{1}}=0.1368991899 \frac{\mathrm{K}}{\mathrm{W}}, \frac{1}{S_{2}}=0.006848732156 \frac{\mathrm{K}}{\mathrm{W}}, \\
\Lambda_{U}=9.96328894 \frac{\mathrm{W}}{\mathrm{K}}, \Lambda_{L}=9.816496019 \frac{\mathrm{W}}{\mathrm{K}} .
\end{gathered}
$$

If we approximate $\Lambda$, the mean value of $\Lambda_{U}$ and $\Lambda_{L}$ then the relative error is less than $0.7421 \%$.

\section{Conclusions}

Upper and lower bounds for the heat flux in nonhomogeneous circular bars of variable diameter are presented. Thermal properties may depend on the radial and axial coordinates. The axisymmetric nonhomogenity considered also includes those cases, when the bar is a composite of different homogeneous materials, so that the thermal conductivity and surface conductivity are piecewise constants. The discontinuities of the thermal properties should not affect the presented analysis. Here we note that for a compound bar the function $F=F(r, z)$ is continuous on the whole meridian section and its normal derivative computed on the curves which separate the different parts of meridian section may have jumps. Normal component of $\boldsymbol{b}$ remains continuous and the tangential component of $\boldsymbol{b}$ may have jumps across the common boundary curves of different phases. Equations of Fourier's theory of steady-state heat conduction are used to formulate the field equations and boundary conditions of the heat transfer 
problem analyzed. Examples illustrate the applications of the bounding formulae derived. The Rayleigh-Ritz method and finite element formulation give possibilities to improve the presented estimation of heat flux in circular bars of variable diameter.

Acknowledgement. The described study was carried out as part of the EFOP-3.6.1-162016-00011 "Younger and Renewing University - Innovative Knowledge City - institutional development of the University of Miskolc aiming at intelligent specialization" project implemented in the framework of the Szechenyi 2020 program. The realization of this project is supported by the European Union, co-financed by the European Social Fund and supported by the National Research, Development and Innovation Office - NKFIH, K115701.

\section{REFERENCES}

1. I. Ecsedi. "A method of estimating the rate of heat flow." Alkalmazott Matematikai Lapok 5(3-4), (1980). In Hungarian, with English summary, pp. 241-247.

2. I. Ecsedi. "The investigation of a problem of heat transfer." Alkalmazott Matematikai Lapok 6(3-4), (1981). In Hungarian, with English summary, pp. 337-344.

3. I. Ecsedi. "Bounds for the heat transfer coefficient." Acta Technica Academiae Scientiarum Hungaricae 95(1-4) (1982), pp. 21-31.

4. I. Ecsedi. "Bounds for the effective heat conduction coefficient." Mechanics Research Communications 29(2-3) (2002), pp. 189-193. DOI: 10.1016/S00936413(02)00238-0.

5. I. Ecsedi. "Mean value and bounding formulae for heat conduction problem." Archive of Mechanics 29(2-3) (2002), pp. 189-197.

6. R. Wojnar. "Upper and lower bounds on heat flux." Journal of Thermal Stresses 21(3-4) (1998), pp. 381-403. DOI: 10.1080/01495739808956153

7. M. Jakob. Heat Transfer. Vol. Vol. I. New York: Wiley, 1949.

8. H. S. Carslaw and J. C. Jaeger. Conduction of Heat in Solids. Oxford: Clarendon Press Oxford, 1986. DOI: 10.1007/978-1-4939-2565-0_2.

9. N. M. Ozisik. Boundary Value Problems of Heat Conduction. New York: Dover Publication, 1989.

10. J. N. Reddy. Energy and Variational Methods in Applied Mechanics. New York: Wiley, 1984.

11. O. C. Zienkiewicz and Y. K. Cheung. The Finite Element Method in Structural and Continuum Mechanics. New York: McGraw-Hill, 1970. 